\title{
Une vue d'ensemble des problèmes concernant l'alerte hydraulique causée par le barrage naturel et par le lac qui s'est formé par suite de l'éboulement de Val Pola
}

\author{
P. Bertacchi, M. Fanelli \\ ENEL, Milano, Italie \\ U. Maione \\ Commission de la Valteline, Sondrio, Italie
}

\begin{abstract}
Ce mémoire montre comment les moyens physiques et mathématiques ont été utilisés pour faire face aux problèmes hydrauliques qui se sont posés lors d'une alerte causée par un glissement de terrain catastrophique qui a créé un barrage et un lac naturels. Les problèmes examinés étaient les suivants: le procédé de formation de voies d'eau causées par le déversement du barrage, le laminage de la crue aval, la percolation à travers le barrage, l'onde éventuellement causée par un glissement de terrain ultérieur. La comparaison finale avec les événements réels a donné une indication sur l'efficacité de l'approche et des moyens choisis.
\end{abstract}

\section{Introduction}

Dans les premières heures de la matinée du 28 juillet 1987 , un important éboulement (plus de $36 \times 10^{6}$ mètres cubes) s'est produit dans la Val Pola à la Valtellina et a barré la rivière Adda à environ 40 kilomètres au nord de Sondrio, en formant un barrage naturel d'environ cent vingt mètres de haut et de deux mille cinq cents mètres de long (voir à ce sujet les figures I et 2, page suiv.). Deux villages ont été détruits et plusieurs autres maisons ont été submergées ; 27 personnes ont péri.
Un lac s'est formé par suite du barrage de la rivière ; dans les jours suivants, le niveau de l'eau est monté : le volume du lac après un mois a été évalué à $20 \times 10^{6}$ mètres cubes.

Le Centre de Recherche Hydraulique et Structurelle (CRIS) de la Direction des Etudes et des Recherches de l'ENEL a été immédiatement chargé par la Commission Technico-Scientifique pour la Valtellina, mise en place par le Département de la Protection Civile, d'entreprendre des études sur les aspects hydrauliques de cette situation délicate.

An overall view of the problems concerning

the hydraulic alert caused by the natural dam and the lake formed after the landslide of Val Pola.

This dissertation shows physical and mathematical means were used to counter the hydraulic problems that were caused at the time of an alert caused by a catastrophic landslide that created a natural dam and lake. The problems examined were the following : the procedure needed to form water channels caused by the overflowing of the dam, drafting of the high water down river, percolation through the dam, the wave possibly caused by a later landslide. The final comparison with real events gave an idea of the efficiency of the means chosen and the overall approach. 

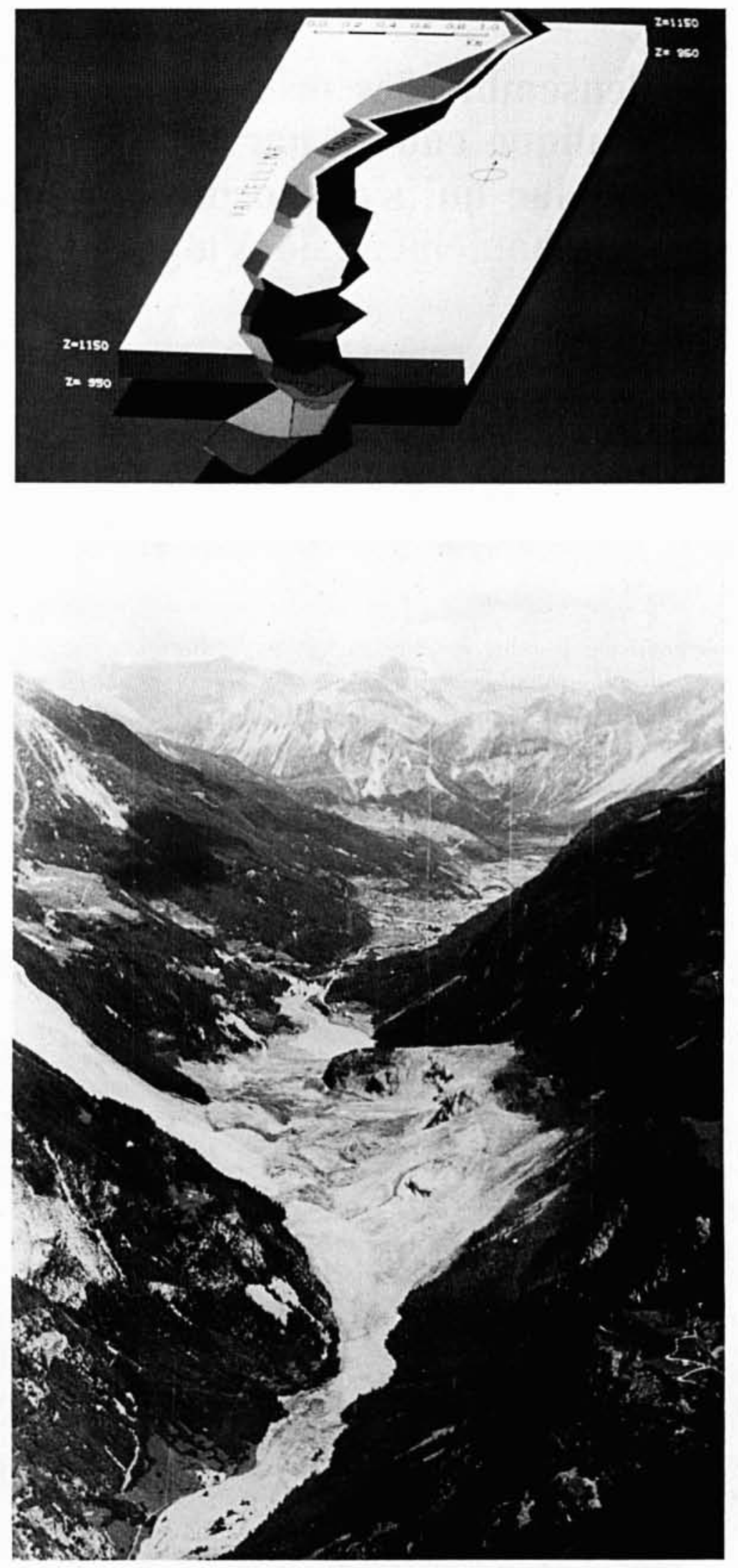

1. Vue d'ensemble du glissement de masse et de la vallée en haut: dessin isométrique en bas: photo du 29.07.1987. 


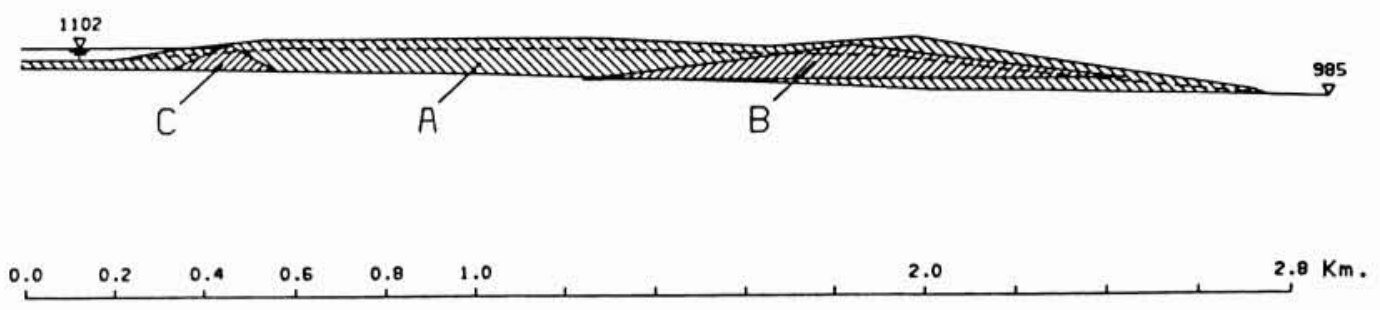

2. Coupe longitudinale du corps du barrage (A).

Par comparaison: la coupe représentée dans le modèle mathématique de formation des voies d'eau (B) et la coupe typique d'un barrage en enrochements (C).

Le premier problème fut d'examiner si le déversement par dessus le barrage naturel formé par l'éboulement aurait pu causer une érosion rapide de la crête et entraîner l'écroulement du barrage, en provoquant une crue catastrophique qui aurait complètement inondé la vallée habitée qui se trouve à l'aval.

Pendant les jours qui ont suivi immédiatement l'éboulement, ce problème a été étudiẻ par des méthodes numériques, en schématisant le barrage naturel comme une masse triangulaire dont les pentes à l'amont et à l'aval étaient égales entre elles et évaluées à $12,5 \%$.

Dans l'hypothèse de la formation d'une brèche par érosion régressive avec l'abaissement résultant du seuil) on a noté que la pente à l'aval avait tendance à se stabiliser. En l'absence de données relatives à la granulométrie du matériel, on a effectué une étude paramétrique avec diffërentes hypothèses sur la dimension des grains.

De la sorte, il a été possible d'obtenir toujours au moyen de méthodes numériques une première évaluation des valeurs maximales du débit de décharge et du temps auquel ce phénomène prend sa valeur maximale. En outre on a estimé les hauteurs et les vitesses des ondes de crue ou ondes de submersion, ainsi que les zones éventuelles submergées à l'aval.

Par ailleurs, deux autres problèmes ont été traités par des modèles mathématiques :

— l'évaluation de l'onde qui aurait pu être causée par un éventuel éboulement ultérieur dans le nouveau lac ;

- la vérification du phénomène de filtration à travers le corps du barrage.

Dans l'intervalle, on a recueilli plusieurs données relatives tant à la forme du barrage naturel qu'aux dimensions du matériau (bien que, dans un certain sens, la granulométrie réelle soit encore inconnue).

Pour mieux reproduire la situation réelle en tenant compte de la morphologie compliquée du corps de ce barrage, il a été décidé de construire un modèle physique hydraulique dans les buts suivants :
1) étudier les conséquences du déversement et, en particulier, l'entraînement du matériau de l'éboulement ; 2) trouver quelques solutions techniques pour diminuer le risque d'une érosion soudaine du corps de ce barrage.

Le modèle hydraulique (dans l'échelle géométrique réduite de $1: 250$ ) a reproduit 8,5 kilomètres de vallée.

On a supposé que le matériau d'éboulement avait une granulométrie moyenne de trente centimètres (plus petite que dans la réalité) et la reproduction a été réalisée dans le modèle en utilisant un matériau granulaire.

Ce modèle physique a été projeté, construit et étalonné en l'espace de 15 jours, étant donné la nécessité d'obtenir une réponse en des temps particulièrement brefs. Le modèle a été terminé avant le remplissage complet du lac.

Les essais effectués sur le modèle, simulant la situation sans perturbation, montrent que le déversement par-dessus le corps du barrage se serait produit sans danger d'éboulement ; de plus, d'autres essais ont montré qu'en abaissant le niveau du seuil et en creusant une section de canal profilé (avec une pente modérée de $2 \%$ ) on aurait obtenu une réduction importante de la valeur maximale du débit à la sortic.

Les résultats des modèles mathématiques et du modèle physique ont été communiqués immédiatement aux Autorités responsables au fur et à mesure qu'ils ont été disponibles, et, en même temps, au Centre des "nouvelles données " d'entrée ont été considérées pour permettre des recherches ultérieures, voir figure 3 (page suiv.). Un mois exactement après l'éboulement, le lac était presque plein et les Autorités responsables ont décidé de faire évacuer environ vingt mille personnes par raison de sécurité, et de procéder à un déversement "contrôlé " en augmentant artificiellement l'apport du fleuve Adda dans le lac au moyen d'un écoulement à l'amont (20-30 mètres cubes/seconde) à partir d'une installation hydroélectrique de l'AEM (Société Electrique Municipale).

Nous donnons, dans les paragraphes qui suivent, de plus amples détails concernant les modèles mathématiques et le modèle physique : nous illustrons, dans le paragraphe des conclusions, des comparaisons entre les deux types de modèles et avec la réalité. 


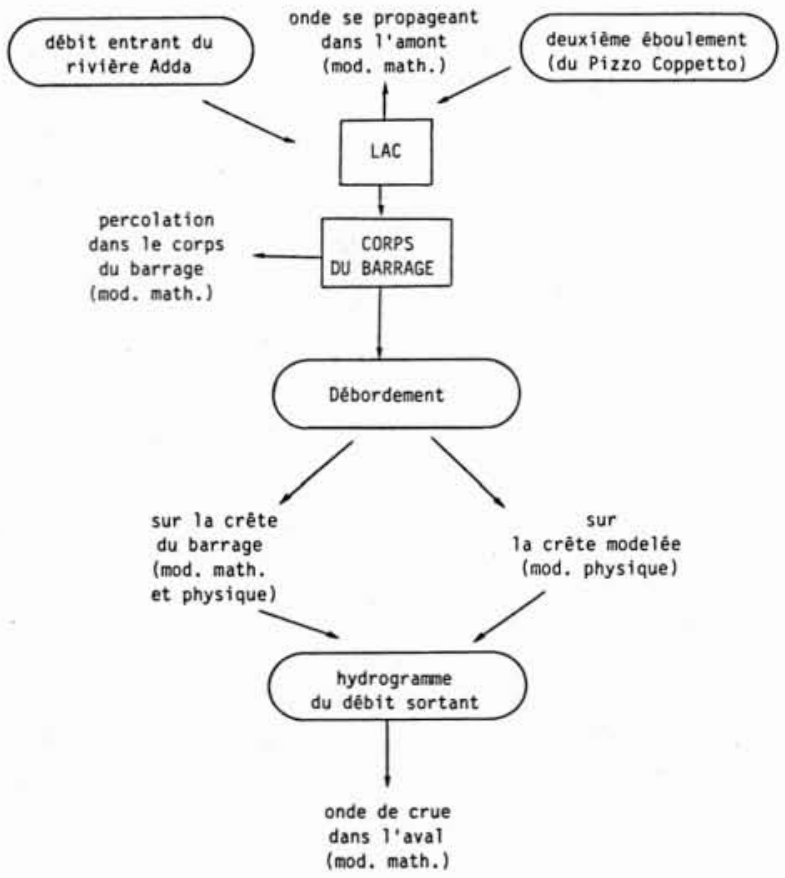

3. Problème et moyens d'étude.

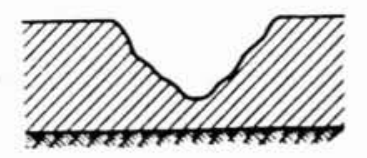

A

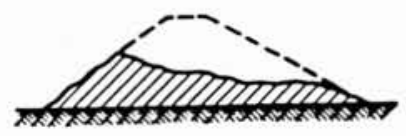

B

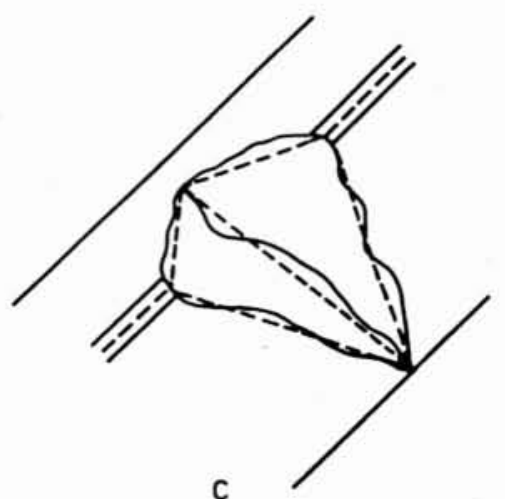

4. Modèle mathématique de l'érosion de l'éboulement: géométrie de la brèche idéalisée.

(A) vue frontale (d'après T.C. MacDonald et al.)

(B) Section transversale de la digue (d'après S.P. Chee)

(C) vue en perspective

\section{Modèle mathématique du phénomène d'érosion causé par le déversement}

Pour évaluer les conséquences possibles d'un déversement par-dessus le barrage, nous avons utilisé un modèle mathématique capable de reproduire le développement d'une brèche dans une digue en terre [1]. Nous avons tenu compte dans le modèle tant des analyses effectuées par MacDonald T.C. et par d'autres auteurs [2] sur un certain nombre d'effondrements de barrage que des résultats expérimentaux de Chee S.P. [3] sur des déversoirs susceptibles d'être érodés.

Le modèle mathématique se base donc sur l'hypothèse que l'érosion et les processus de modification causés par l'écoulement de l'eau à travers la brèche ont lieu suivant un schéma géométrique idéal de brèche illustré par la figure 4.

La vidange du bassin est calculée d'après la formule d'un déversoir à large seuil adaptée à un déversoir à profil triangulaire.

Le débit sur la brèche, dans les conditions dynamiques, est considéré comme un débit presque stationnaire et son évolution est calculée en se basant sur l'équation de Bernoulli, les pertes énergétiques étant estimées par la formule de Strickler.

L'évaluation de l'érosion du barrage est reliée à la capacité maximale de transport du débit au pied de la brèche ; cette capacité de transport est calculée suivant la formule
d'Engelund, qui, étant une corrélation de transport total du matériau, comprend tant le transport du matériau charrié sur le fond que celui des matières en suspension. Finalement, la vidange du lac est simulée avec la méthode du "storage routing".

Le modèle avait été appliqué précédemment à la simulation de certains cas de rupture de barrages en terre ; dans la plupart des cas, les résultats ont montré un accord raisonnable avec les observations dans la nature.

Par ailleurs, l'ordre de grandeur des concentrations du débit solide données par la formule d'Engelund s'est montré en bon accord avec les observations expérimentales de S.P. Chee [3].

L'érosion du barrage naturel de Val Pola a été étudiée en supposant différentes valeurs du diamètre significatif du matériau granulaire et du débit arrivant au lac à l'amont ; nous avons étudié également des situations dans lesquelles la crête était plus ou moins abaissée. Il en est résulté que l'abaissement du seuil avait une influence positive prononcée en ce sens que le débit maximal à la sortie en aval diminuait considérablement.

Les résultats obtenus, en terme du volume de matériau enlevé durant le processus de l'érosion, du temps de développement de la brèche et des valeurs maximales des débits à la sortie, ont été représentés graphiquement sous la forme adoptée par MacDonald et autres [2]. Nous avons reporté dans la figure 5 les diagrammes originaux sur lesquels sont superposés les points correspondant aux essais sur le modèle mathématique réalisé par le CRIS. 

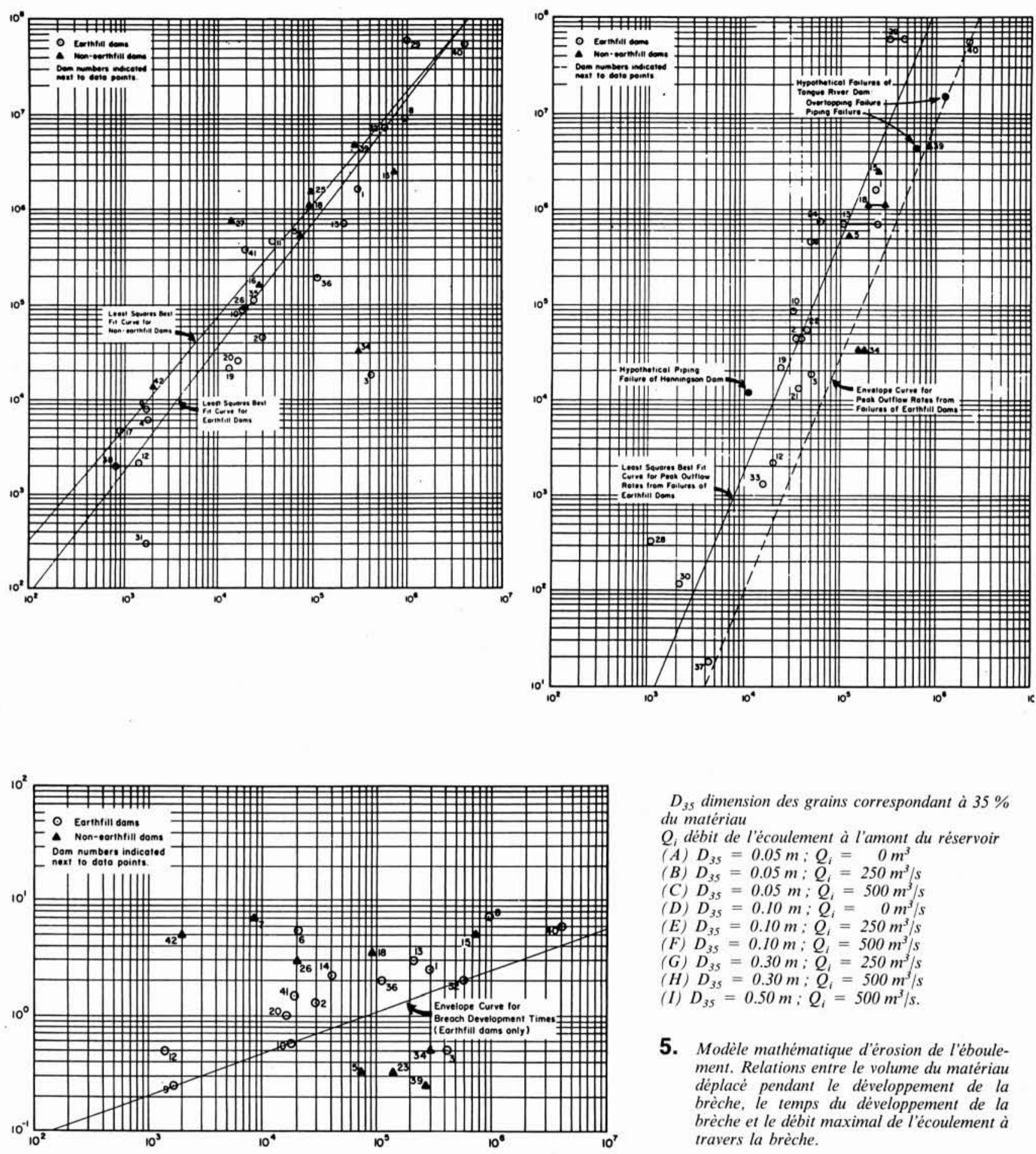

$D_{35}$ dimension des grains correspondant à $35 \%$ du matériau

$Q_{i}$ débit de l'écoulement à l'amont du réservoir

(A) $D_{35}=0.05 \mathrm{~m}: Q_{i}=0 \mathrm{~m}^{3}$

(B) $D_{35}=0.05 \mathrm{~m} ; Q_{i}=250 \mathrm{~m}^{3} / \mathrm{s}$

(C) $D_{35}=0.05 \mathrm{~m} ; Q_{i}=500 \mathrm{~m}^{3} / \mathrm{s}$

(D) $D_{35}=0.10 \mathrm{~m} ; Q_{i}=0 \mathrm{~m}^{3} / \mathrm{s}$

(E) $D_{35}=0.10 \mathrm{~m}: Q_{i}=250 \mathrm{~m}^{3} / \mathrm{s}$

(F) $D_{3 s}=0.10 \mathrm{~m} ; Q_{i}=500 \mathrm{~m}^{3} \mathrm{~s}$

(G) $D_{35}=0.30 \mathrm{~m} ; Q_{i}=250 \mathrm{~m}^{3} / \mathrm{s}$

(H) $D_{35}=0.30 \mathrm{~m} ; Q_{i}=500 \mathrm{~m}^{3} / \mathrm{s}$

(I) $D_{35}=0.50 \mathrm{~m} ; Q_{i}=500 \mathrm{~m}^{3} / \mathrm{s}$.

5. Modèle mathématique d'érosion de l'éboulement. Relations entre le volume du matériau déplacé pendant le développement de la brèche, le temps du développement de la brèche et le débit maximal de l'écoulement à travers la brèche.

Si l'on examine ces figures, il est possible d'observer que, suivant le modèle, l'érosion de l'éboulement de Val Pola se produirait plus graduellement et avec des débits maximaux inférieurs par comparaison avec les barrages en terre; ceci paraît raisonnable, vu le plus grand volume, les pentes plus douces et la hauteur relativement modeste du barrage naturel. La situation effective s'est montrée par la suite, encore plus favorable, vu les hypothèses conservatives adoptées pour définir la section transversale employée dans le modèle mathématique. 


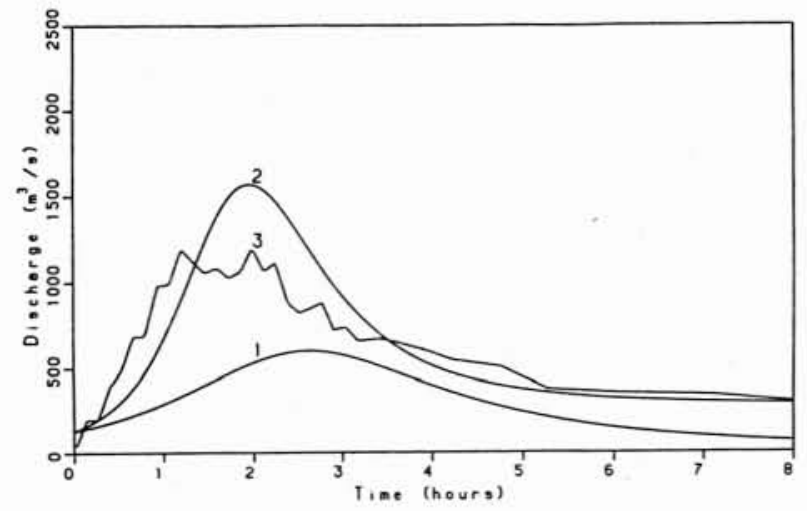

6. Hydrogramme du débit conséquent à l'érosion par déversement de la digue de Val Pola : comparaison entre le modèle physique et le modèle mathématique dans le cas de seuil non modifié. (1) modèle mathématique avec $D_{35}=0.10 \mathrm{~m}$ et $Q_{i}=0 \mathrm{~m}^{3} / \mathrm{s}$ (2) modèle mathématique avec $D_{35}=0.10 \mathrm{met} Q_{i}=250 \mathrm{~m}^{3} / \mathrm{s}$ (3) modèle physique avec $D_{35}=0.18 \mathrm{~m}$ et $Q_{i}=50 \mathrm{~m}^{3} / \mathrm{s}$.

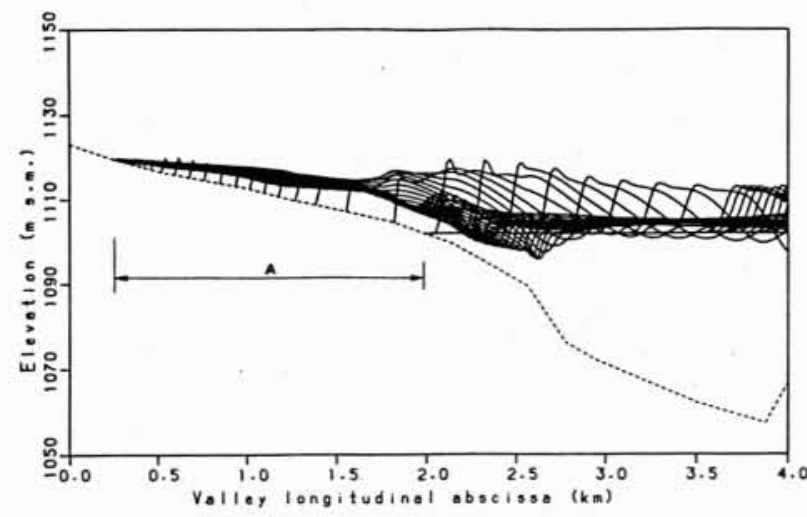

7. Réservoir de Val Pola : profils instantanés de l'onde produite par un nouvel éboulement.

(A) longueur de l'intrusion de la vague à l'extrémité amont du lac

\section{Modèle mathématique de la propagation de l'onde} de crue ou onde de submersion à l'aval

La modélisation mathématique de l'érosion du barrage de Val Pola a montré, que, si on laisse inchangée la côte de la crête du barrage et si l'on considère une granulométrie plutôt fine du matériau, certaines crues dans le bassin à l'amont pouvaient produire des inondations très graves à l'aval. Pour calculer les caractéristiques de ces ondes de crue, on a utilisé le modèle numérique STREAM, développé par le CRIS pour le calcul des ondes de crue qui suivent l'écoulement des digues [4]. Ce modèle se base sur la formulation faible des équations de De Saint Venant qui autorise la simulation des ondes à front raide, et l'on emploie une grille de calcul mobile facilitant la simulation de la propagation des ondes sur fond sec.
De cette façon, dans les différentes hypothèses considérées, on a calculé pour les sections de la vallée qui se trouvent à l'aval du barrage - sur une longueur de quarante kilomètres - les hauteurs moyennes atteintes par l'eau, les vitesses maximales du courant et les temps d'arrivée de l'onde de crue.

\section{Modèle mathématique de la vague causée par un nouvel éboulement possible, le lac étant plein}

Outre à la propagation de l'onde de crue, une autre application intéressante du modèle STREAM concerne la propagation, dans le lac formé par le barrage de Val Pola, d'une onde de translation causée par un éboulement ultérieur.

D'après les informations fournies par les géologues, on pouvait supposer que le volume de l'éboulement était de $2 \times 10^{6}$ mètres cubes avec un temps de chute de vingt secondes. Pour simuler les effets produits par l'éboulement sur les eaux accumulées, on a supposé dans le calcul un mouvement vers l'amont "à piston ", à partir de l'aval, de l'extrémité du réservoir, déplaçant un volume d'eau égal à celui du matériau de l'éboulement qui s'immerge dans le lac.

La figure 7 montre les profils instantanés de l'onde. Sur la base de ces simulations (conduites aussi en faisant varier la section d'immersion de l'éboulement, le volume et le temps de chute) on a pu conclure que l'onde de translation ne serait pas remontée vers l'amont (dans la direction de Bormio) de plus de 1,5-2 kilomètres, que la hauteur maximale du front de l'onde à son arrivée à l'amont du lac aurait été de l'ordre de 10 mètres et que les volumes d'eau débordant et entraînés vers l'aval auraient été relativement faibles (quelques centaines de milliers de mètres cubes).

\section{Modèle mathématique du mouvement de filtration à travers le barrage naturel}

On a construit un modèle mathématique plan (dans un plan vertical) à éléments finis qui schématise sous une forme bidimensionnelle la masse éboulée, afin d'évaluer les débits de filtration qui pouvaient traverser cette masse poreuse et les dangers de filtration rapide (effet de renard).

Etant donné que le but était d'évaluer les ordres de grandeur et que les informations sur le matériau étaient très approximatives, on n'a pas estimé utile de faire recours aux modèles mathématiques tridimensionnels disponibles également auprès du CRIS.

Le modèle mathématique utilisé se base sur l'emploi du programme EFFIGE-2D développé par le CRIS [5]. Il se base sur la méthode des Eléments Finis et il peut simuler des mouvements de filtration à surface libre tant stationnaires que non stationnaires. Différents cas ont été étudiés, 
en faisant varier le coefficient de perméabilité du matériau et la cote de la retenue à l'amont [6].

Les conclusions de cette étude, par rapport à ce qui a été observé sur place, ont permis de conclure que le coefficient de filtration moyen de l'amas devait être inférieur a $10^{-2} \mathrm{~ms}^{-1}$, que les risques de filtration rapide (effet de renard) n'existaient pas et que les débits de filtration s'avéraient négligeables en comparaison à ceux arrivant de lamont.

\section{Modèle hydraulique du déversement du barrage naturel}

Le modèle, construit en dix jours, a quatre mètres de large et quarante mètres de long. Il reproduit en ciment lissé environ 8,5 kilomètres de la topographie de la Valtellina, ainsi qu'elle résultait des cartes antérieures à l'éboulement du 28 juillet 1987. Sur ce fond rigide on a successivement reproduit, avec un matériau granulaire convenable (voir plus loin), la masse de l'éboulement, en recourant à des levés aérophotogrammétriques disponibles peu de jours après que l'éboulement se soit produit.

L'échelle de similitude géométrique est $l_{r}=1: 250$; par conséquent, étant donné que l'on adopte la similitude de Froude, l'échelle des temps est $t_{r}=1: 15,8$. Le barrage formé par l'éboulement est reproduit en matériau granulaire non cohésif formé par un mélange de sable siliceux et de calcaire granulaire (1-2 m). La densité relative des grains dans l'eau est la même que celle des débris de l'éboulement; en supposant la similitude de transport basée sur le paramètre de Shields [3] les échelles du diamètre des grains et des temps d'érosion (transport du matériau charrié sur le fond) sont respectivement :

$d_{r}=I_{r}=1: 250$,

$t_{e}=t_{r}=1: 15,8$

(pourvu que le mouvement de l'eau soit également turbulent sur le modèle, ce qui impose une limite inférieure aux débits à représenter sur le modèle).

La plupart des essais ont été conduits en employant un matériau dont la granulométrie correspond à un diamètre moyen réel $d_{50}=261 \mathrm{~mm}$ [7].

La technique de la décantation pluviale [8] a été employée pour former le barrage sur la surface rigide du fond de la vallée. Cette méthode permet d'obtenir la densité apparente maximale du matériau, ce qui assure la répétibilité des essais.

La morphologie de la masse a été obtenue en disposant, au cours de la décantation, des formes horizontales successives de contreplaqué qui reproduisaient les courbes de niveau résultant des levés aérophotogrammétriques de l'éboulement.

L'ensemble des méthodes employées tend à réduire au minimum la variabilité due au travail manuel des opérateurs durant la formation du barrage, afin de pouvoir assurer le plus possible la répétibilité des essais.

Il est important de mettre en évidence que la densité volumétrique du barrage, la granulométrie adoptée et l'uniformité du matériau de modélisation ne correspondent pas à la composition effective et à la structure interne de la masse, certainement hétérogénes et en grande partie inconnues au moment de la réalisation du modèle.

Ces caractéristiques doivent être considérées comme des hypothẻses de base de l'expérimentation, plutôt idéalisées à titre de précaution, capables de réaliser une masse d'éboulement que l'on peut facilement former plusieurs fois sur le modèle et qui peut facilement être comparé aux modèles théoriques employés dans les simulations numériques.

En vingt jours (y compris la période de construction et d'étalonnage) nous avons effectué huit essais en simulant le déversement de la masse tant dans sa conformation naturelle qu'après différentes interventions modifiant son sommet : en particulier, un canal de décharge a été creusé et nous avons comparé les effets de différentes profondeurs, largeurs et formes du canal en question. Nous avons également enquêté sur l'influence des débits d'entrée dans le lac. Nous avons comparé les hydrogrammes respectifs de crue à l'aval de la masse et les mécanismes ainsi que les temps d'érosion relatifs, afin de choisir la meilleure solution technique de déversement parmi celles proposées par la Commission créée par la Protection Civile. Nous avons constaté que l'abaissement du seuil et la présence d'un canal régulier à pente modérée $(2 \%)$ produisaient une réduction considérable des débits maximaux à l'aval.

Les comparaisons effectuées avec les résultats des modèles mathématiques (voir le paragraphe 7) et avec le développement du déversement réel (voir fig. 6) ont confirmé les caractéristiques indiquées par le modèle physique: ceci montre que les hypothèses simplificatrices adoptées conservent les paramètres physiques caractéristiques du processus effectif d'érosion.

\section{Conclusions}

Ce rapport illustre les moyens de recherche mathématiques et physiques qui ont été employés au CRIS, en rapport étroit avec la Commission technico-scientifique pour la Valtellina nommée par le Département de la protection civile, pour faire face à différents problèmes hydrauliques qui se sont présentés à la suite du cas d'urgence de l'éboulement (voir fig. 3).

On a effectué des vérifications croisées entre les résultats obtenus par différents moyens, afin de s'assurer de la cohérence des hypothèses de départ et des résultats; nous donnons ci-dessous un exemple de comparaison possible.

Dans l'essai $\mathrm{n}^{\circ} 3$ effectué sur le modèle hydraulique physique (en date du 21 août 1987) on a employé un matériau granulaire avec $D_{35}=18$ centimètres $e D_{65}=$ 32 centimètres; le débit à l'arrivée dans la retenue était fixé à 50 mètres cubes/seconde ${ }^{-1}$ (valeur en accord avec la situation réelle). Parmi les différents cas analysés avec le modèle mathématique d'érosion avant le $1^{\text {er }}$ août 1987 , il $y$ en a deux qui encadrent celui de l'essai $n^{\circ} 3$. Dans les deux cas on avait $D_{35}=10 \mathrm{~cm}, D_{65}=25 \mathrm{~cm}$, et la profon- 
deur initiale de la brèche était égale à 5 mètres; le débit à l'arrivée à la retenue était nul dans un cas et égal à 250 mètres cubes/seconde dans l'autre cas.

La comparaison entre les hydrogrammes de crue à l'aval fournis par le modèle physique pour l'essai $n^{\circ} 3$ et par le modèle mathématique pour les deux cas cités ci-dessus est illustrée à la figure 6. De cette comparaison nous pouvons conclure que les deux moyens différents de recherche fournissent des résultats concordant de façon satisfaisante.

Les comparaisons avec le développement des événements réels fournissaient elles aussì des résultats satisfaisants. Nous avons déjà parlé des informations concernant le coefficient de perméabilité de la masse (voir le paragraphe 5). La comparaison des processus de déversement dans le modèle hydraulique physique et dans la réalité est plus spectaculaire : l'écoulement des eaux s'est avéré très similaire à ce qui a été vu (et enregistré) sur le modèle, avec la même morphologie d'érosion, seulement moins intense dans certaines zones, grâce à des concentrations locales — dans la réalité - de rochers de grosses dimensions (non reproduite sur le modèle), voir figure 8.

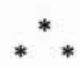

Certaines observations finales permettent de mettre en évidence les caractéristiques les plus saillantes de l'ensemble des enquêtes.

Motifs : Support pour les décisions techniques (et contrôles des solutions choisies), rendu nécessaire par le fait que la sécurité de plus de 20000 personnes était concernée.

Temps : Un peu plus d'un mois s'est écoulé entre l'éboulement et le déversement. Durant cette période, des modèles mathématiques et physiques ont été mis en action.

Les résultats relatifs ont été comparés entre eux. Ils ont été également comparés avec des données relevées dans la réalité.

Les derniers essais sur le modèle physique ont été effectués alors que les excavateurs à benne traînante creusaient le canal de décharge et que le lac était presque plein.

Nous retenons que c'est la première fois que l'on ait employé un modèle physique "en temps réel ".

Vision d'ensemble: Différents problèmes hydrauliques ont été abordés par diverses équipes techniques ayant des compétences différentes, toutes cependant travaillant dans le même centre de recherche (le CRIS). Ceci a permis une très étroite collaboration, un très rapide échange d'informations et le maximum d'efficacité du travail, en rendant disponibles pour les Autorités responsables les réponses nécessaires pour orienter les décisions dans un temps minimal.

\section{Remerciements}

Nous nous devons de citer - sans prétendre être complets - la contribution experte et enthousiaste offerte au cours de cette enquête par les ingénieurs et les chercheurs suivants :

- A. Di Monaco, G. Giuseppetti, P. Molinaro,

R. Rangogni, pour les modèles mathématiques;

- G. Angelico, M. de Gerloni pour le modèle physique ; A. Zaninetti pour les aspects géotechniques de la reproduction simplifiée du corps de l'éboulement.

Nous désirons rappeler la collaboration que nous a assuré l'ISMES en ce qui concerne la fourniture des données topographiques et la participation du personnel technique à l'emploi des modèles mathématiques.

\section{References bibliographiques}

[1] ENEL-DSR-CRIS. - Modèle mathématique de la brèche qui se développe dans un barrage en matériel libre par débordement. Rapport n³444, octobre 1986.

[2] T.C. McDonald, J. LANGRidge-Monopolis. - Breaching characteristics of dam failures (caractéristiques de rupture des digues défectueuses). Journal of Hydraulic Engineering ASCE, Vol. 110, n 5, mai 1984.

[3] S.P. CHEE. - Washout of spillway dams (érosion des digues à déversoir). Channels and Channel Control structures, Proceedings of the First International Conference, Southampton. England, april 1984.

[4] A. Di Monaco, P. Molinaro. - Lagrangian finite element model of dam-break wave on dry bed versus experimental data (modèle d'éléments finis de Lagrange de vague de crue de digue sur un lit sec vis-à-vis des données expérimentales). HYDROSOFT 1984, Proceedings of the First International Conference, Portoroz, Yugoslavia, septembre 1984.

[5] ENEL-DSR-CrIS. - Le code de calcul EFFIGE 2D. Première partie: Théorie, procédés numériques et structure. Deuxième partie : Problèmes d'essai pour la vérification et la qualification en garantie de la qualité. Rapport n. 3316, juillet 1985 e n. 3333, septembre 1985.

[6] ENEL-DSR-CRIS. Digue de Val Pola, étude théorique du mouvement de filtration. Rapport n. 3544, septembre 1987.

[7] ENEL-DSR-CRIS. Modèle hydraulique physique pour l'étude du débordement sur le barrage naturel formé par l'éboulement de Val Pola. Rapport n. 3547, octobre 1987.

[8] R. BelLotri, G. Bızzi et d'autres auteurs. - ENEL approach to the evaluation of the liquefaction potential of sand deposits (méthode de la société ENEL pour l'évaluation du potentiel de liquéfaction des dépôts de sable. ICOLD 13th Congress, New Delhi, 1979). 

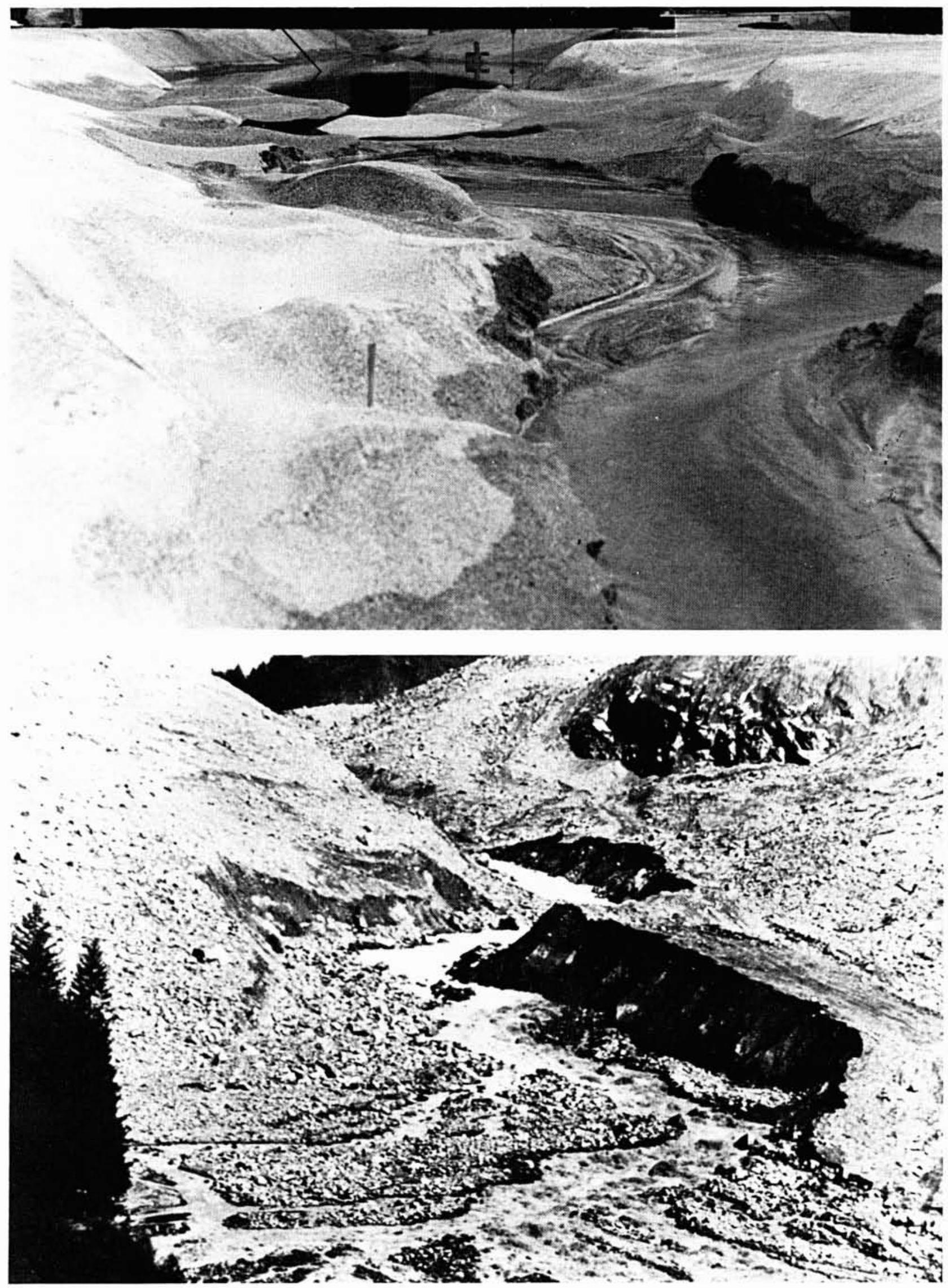

8. L'érosion causée par le déversement (vue d'aval)

en haut dans le modèle hydraulique physique (14.08.87)

en bas dans la réalité (03.09.1987). 
9. Vue d'ensemble du modèle hydraulique physique (vu d'amont).

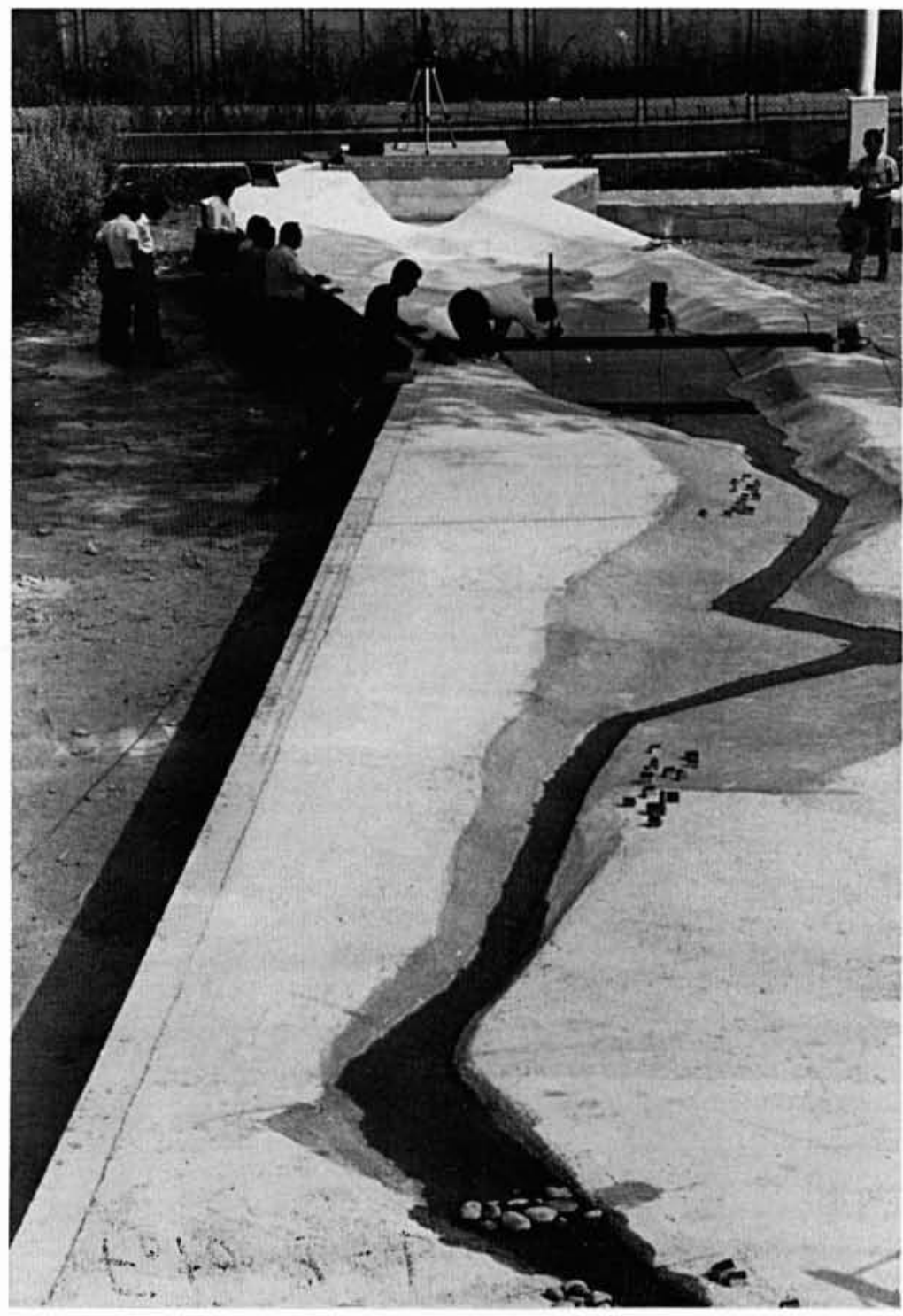

\section{Discussion}

(Société hydrotechnique de France - Nov. 1987)

M. GovBET: Comment a-t-on déterminé les caractéristiques des matériaux à utiliser pour simuler l'éboulement dans le modèle physique? (cohésion, granulométrie, ....).

M. FANELLI: Ceci a constitué une des marges d'incertitude les plus grandes dans cette étude. Nous connaissions le diamètre moyen dans la nature $(30 \mathrm{~cm})$, et nous savions qu'il s'agissait de roches dioritiques. La proportion des fines était de $30 \%$. Nous nous sommes arrangé pour avoir sur le matériau granulaire employé sur le modèle, reproduit à l'échelle, les mêmes diamètres et la même proportion de fines. La porosité était la même, la cohésion nulle. Nous ne savons pas s'il y a de la cohésion dans la réalité, mais elle doit être faible.

$M$. ERLICH : La rapidité avec laquelle vous avez réussi à construire vos modèles reduits est impressionnante, mais je suppose que, grâce aux résultats des simulations numériques, vous avez travaillé tout en sachant avec certitude qu'il n'y avait pas de danger et que le processus était relativement lent?

M. FANELLI: Dans le cadre de cette étude, nous avions les premiers résultats des modèles mathématiques, et l'impression qu'il n'y aurait pas de danger catastrophique. Nous devions démontrer ceci avec un modèle tridimensionnel, le modèle numérique étant bidimensionnel. Les résultats sur le modèle réduit ont été satisfaisants, d'autant plus qu'il n'y a pas eu d'événements supplémentaires catastrophiques.

M. Le Président PICARD: Merci de cet exemple d'aide à la décision technico-politique. Il s'agissait aussi de rassurer les personnes à l'aval.

M. FANELLI: A un certain moment les autorités publiques ont évacué 28000 personnes. La réalisation du modèle physique a permis de prendre la décision de permettre a ces personnes de rentrer chez elles. 